\title{
Teaching Lean with Virtual Reality: Gemba VR
}

\section{Conference Paper}

Author(s):

Netland, Torbjörn (D); Lorenz, Rafael (D); Senoner, Julian

Publication date:

2019-11

Permanent link:

https://doi.org/10.3929/ethz-b-000387713

Rights / license:

In Copyright - Non-Commercial Use Permitted 


\title{
Teaching Lean with Virtual Reality: Gemba VR
}

\author{
Torbjørn H. Netland ${ }^{1}$, Rafael Lorenz ${ }^{1}$, Julian Senoner ${ }^{1}$ \\ ${ }^{1}$ ETH Zurich \\ Department of Management, Technology, and Economics \\ Chair of Production and Operations Management \\ Weinbergstrasse 56/58, 8092 Zürich, Switzerland \\ tnetland@ethz.ch, rafaellorenz@ethz.ch, jsenoner@ethz.ch
}

\begin{abstract}
Lean production is best taught on the factory floor. Yet, in higher education, it is almost exclusively taught in classrooms. We want to keep and proliferate the learning experience of exploring a real factory's "Gemba" and, at the same time, to remove limitations to factory visits. Due to recent developments in virtual reality (VR) technologies, VR offers excellent opportunities to achieve this. In this paper, we present an innovative way to teach lean production with VR. We show how we implemented a solution to let students be immersed in the factories of Toyota, $\mathrm{ABB}$, and other world-renowned companies without having to travel. We also report on our experiences and provide other teachers the information needed to adopt "Gemba VR" in their own teaching.
\end{abstract}

Keywords. teaching lean, lean production, virtual reality, Toyota, Gemba

\section{Introduction}

Training in lean production is most effective at the place it is supposed to be implemented, that is, the "Gemba." The term is Japanese, meaning "the actual place" [1, 2]; for lean production, that means the factory floor. Yet almost all lean training and instruction in the higher education sector takes place in classrooms. There are obvious reasons for this, but in this paper we show that —due to recent advancements in virtual reality (VR) - teachers can bring the Gemba to the classroom. We demonstrate and discuss the incorporation of online virtual environments from real factories in course designs.

Field trips are an effective way of teaching lean in higher education [3], but field trips are generally inefficient. For example, it can be difficult to gain access to factories, and entering all the areas relevant to the class may not be permitted. Factory visits require considerable resources to coordinate and organize, especially for class sizes exceeding 25 students. It can also be difficult to fit factory visits into busy study and teaching schedules. Because field trips are difficult to plan and organize, they are rare learning opportunities for students. What if-rather than the students going to the field - the field could be brought to the students? 
We want to keep and proliferate the learning experience of exploring a real factory environment and, at the same time, remove limitations to field visits. Recent developments in VR technologies offer excellent opportunities to do so. In this paper, we present a teaching innovation that takes advantage of VR to teach lean production. In the next section, we present the technology and concept of "Gemba VR." After that, we draw on our experiences from using this concept in three different courses at ETH Zurich to discuss opportunities and challenges. We also provide advice for teachers who would like to teach with VR.

\section{Introducing Gemba VR}

There is agreement in the pedagogy literature that active learning is superior to passive learning $[4,5]$. For example, the "70-20-10 rule" of learning suggests that managers learn $70 \%$ from on-the-job experiences and challenges, $20 \%$ from what they hear from other people, and only $10 \%$ from classroom training [6]. In light of these findings, it is disappointing that the vast amount of training in lean production takes place in classrooms. While this is obviously true for higher education, it is unfortunately also true for many training programs in companies. This problem is exaggerated when it comes to training in lean production, as it is hard to teach accurately in classrooms. While the introduction to tools and techniques can be covered [7], it is not easy to convey the complexity involved in applying them to real factory setting. It is even harder to teach the important cultural and behavioral elements of lean in the classroom [8].

VR can help mitigate some of the drawbacks of traditional classroom teaching in higher education [9]. VR is an artificial environment presented to the user so that the user experiences it as a close-to-real environment $[10,11]$. The VR environment can be an artificial creation, a digital copy of the real world, or a combination of both. The form of VR that we refer to in this paper is 3-dimensional images and videos that can be explored with a computer screen or wearable VR headsets. By offering the students VRs of real factories, they can actively explore the virtual content guided by assignment questions. We call this idea "Gemba VR." We take advantage of available technologies to show how Gemba VR can be implemented cost-effectively and effortlessly at scale.

Gemba VR consists of hardware, software, and a task assignment, all of which we introduce below.

\subsection{Hardware}

The hardware consists of students' own smartphones and a VR headset. It is anticipated that students have their own smartphones that they can use for this purpose. Figure 1 shows teaching staff using two different VR headsets: The person on the left uses a cardboard headset that cost $\$ 3$, and the person on the right uses a plastic headset that cost $\$ 30$. We have used both in Gemba VR. The cardboard version is cheap enough to hand out freely to all students, while the plastic headsets are reused by other students. More professional VR equipment (e.g., HTC Vive, Oculus Rift) also exist, but these 
technologies are difficult to scale due to use requirements (e.g., space and marking) and costs.
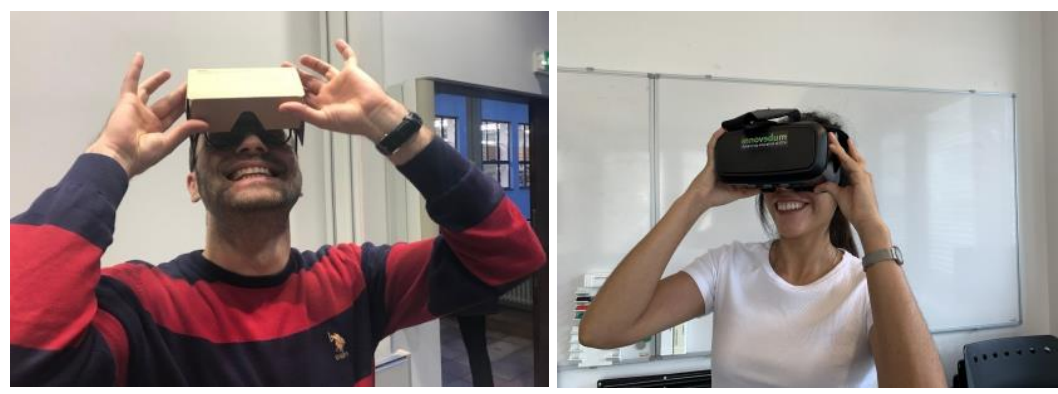

Fig. 1. Teaching staff using a VR cardboard headset (left) and a plastic headset (right).

\subsection{Software}

We use software that is freely available online or in mobile applications (apps). Table 1 provides a list of exemplar software that can be used. YouTube contains a number of 360-degree videos of factories. In addition, more and more companies are launching VR apps in which users can explore factories freely at their own pace. For example, since 2017, the ABB Group has offered VR environments of some of their factories through an app available in major app stores. The app consists of 360-degree pictures and videos from five factories blended with virtual instructions and information. In the app, students can visit a number of predefined areas in the factories in any order they like. In our course, we have chosen to use the ABB app and a Toyota YouTube video, as the richness of this software fit well with our specific learning objectives.

Table 1. Examples of available VR content that can be used to teach lean production.

\begin{tabular}{llllll}
\hline Company & Location(s) & Title & Format & $\begin{array}{l}\text { Dura- } \\
\text { tion } \\
\text { (min) }\end{array}$ & Access links \\
\hline ABB & CH (2), DE, & ABB 360 VR tours & App & N/A & $\begin{array}{l}\text { AppStore } \\
\text { GooglePlay }\end{array}$ \\
& FIN (2) & Ashington VR & App & N/A & GooglePlay \\
AkzoNobel & UK & Toyota VR / 360 Factory Tour & 360 video & 2.56 & YouTube \\
Toyota & FRA & Inside a Gas Turbine Factory & 360 video & 3.50 & YouTube \\
GE & US & See where Tesla makes its cars & 360 video & 2.06 & YouTube \\
Tesla & US & Explore VR Plant & 360 video & 4.43 & YouTube \\
Hyundai & KOR & VR Factory Tour From Above & 360 video & 2.01 & YouTube \\
Niftylift & UK & TVS - 360 Factory Tour & 360 video & 2.07 & YouTube \\
TVS & IND & Würth Elektronik Factory Tour & 360 video & 2.28 & YouTube \\
Würth & DE & & & &
\end{tabular}


360-degree videos and VR apps have pros and cons. Among the pros are that the apps are easier to navigate and allow the user to visit different locations in the factory at his or her own speed. They can also be operated seamlessly with most VR headsets. Among the cons are that the virtual rooms are often static or only show short videos on repeat. The apps are therefore generally unsuitable for showing the material flow in the factory. The videos (e.g., the Toyota video listed in Table 1), however, are excellent for showing material flow. The drawback of videos is that they are often played at a high speed, which makes it hard to absorb all their details. This issue can be mitigated by pausing the videos or manually setting the play speed at a fraction of the standard (e.g., in YouTube, set the "playback speed" to 0.25 ). While a slower play speed makes it much easier to follow, any audio material will be practically unusable and should be muted.

By using both VR apps and 360-degree videos in our courses, we take advantage of the strengths of both. Figure 2 shows two examples from the apps we use in class. The left picture is a snapshot of a running 360-degree video that follows the assembly of a forklift from start to end in a Toyota factory. The right picture is a repeated 360-degree video that shows how the operator uses a machine in an $\mathrm{ABB}$ electronics assembly factory. In the right picture, the information icon (the red " $i$ " in a white circle) contains blended information that can be clicked and read. In both the video and the app, the user can explore the assembly halls by turning the headsets in 360 degrees, or if navigated on a screen, the user can use the navigation panel to look around.
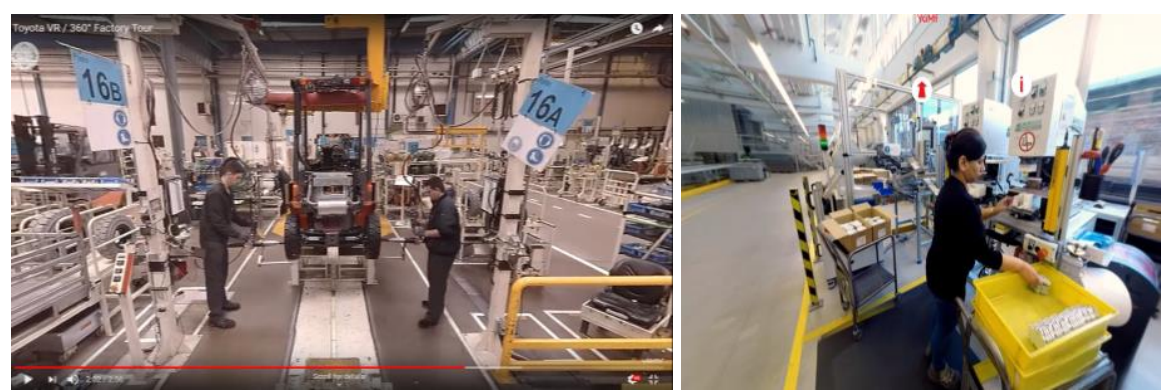

Fig. 2. Snapshots from VR environments in Toyota (left) and ABB (right).

\subsection{Assignment}

We implement Gemba VR as a work assignment in the courses. The assignment requires students to solve questions related to the learning objectives of the course. To answer the questions, the students have to visit the virtual environments. We ask the students to form groups of 3-5 people to discuss the content, but the assignment could also be given as individual coursework. Every student is encouraged to first solve the questions individually before meeting and discussing in their groups. The assignment lasts about three weeks. The student groups must hand in group reports answering all the assignment questions and also prepare short presentations containing their answers to the questions. We dedicate a class to present and discuss randomly drawn group reports. 
Table 2 lists the questions we ask in the 2019 fall semester course. We separate three different types of questions (seek-and-find, explore-and-think, and compare-and-analyze), which increase in difficulty as the students progress with the assignment. Other teachers can and should adapt the assignment questions to fit their own course's learning objectives.

Table 2. Assignment questions.

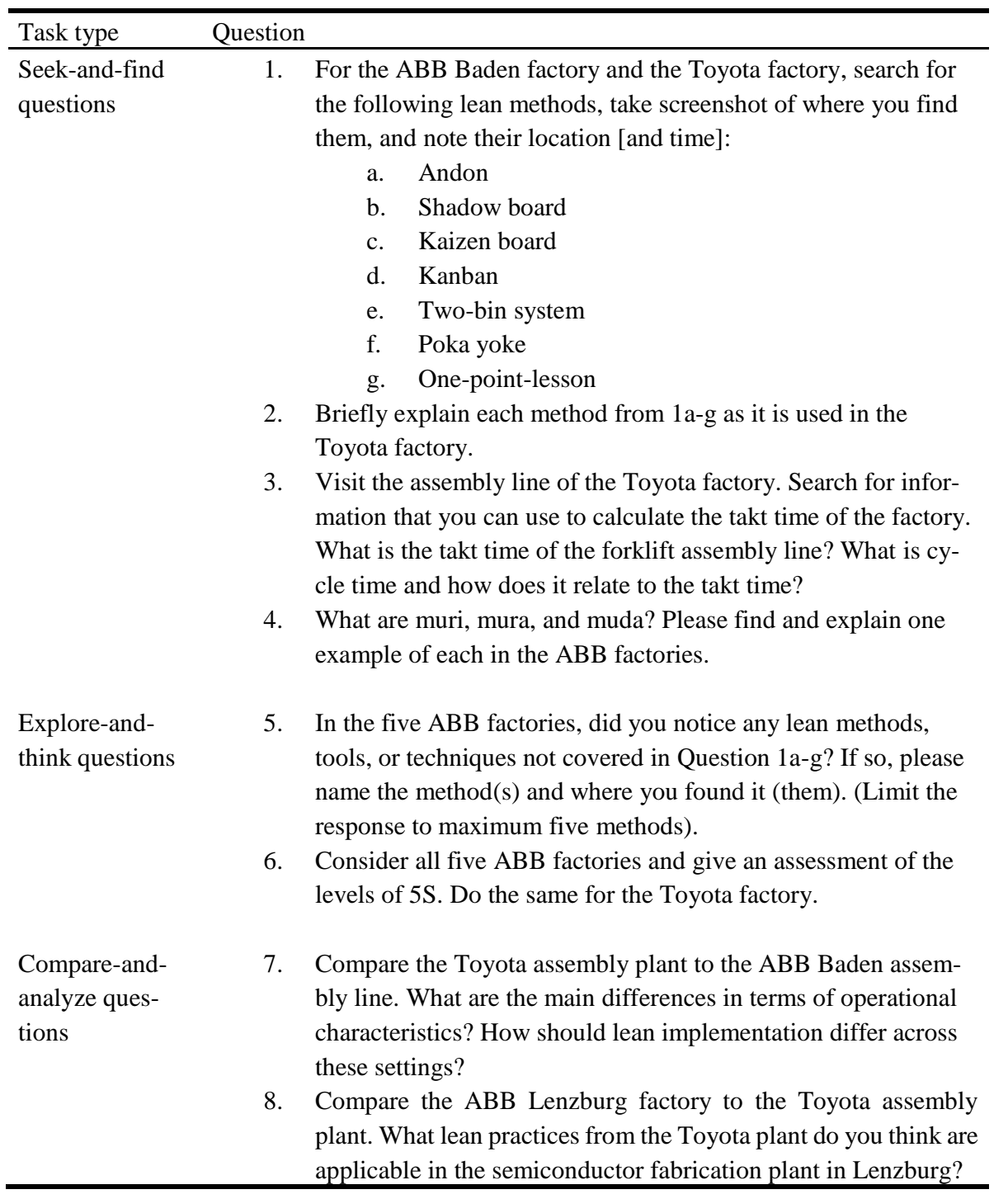




\section{Experiences from teaching with Gemba VR}

Our experience with teaching lean with VR is that students generally appreciate the innovation. In particular, they report that they enjoy the opportunity to be immersed in a real factory. Figure 2 shows a group of Master of Business Administration (MBA) students solving questions with the use of VR. One of these students summarized the assignment as follows: "The VR experience was tremendous. It was a great way to get a good insight into production facilities and to start analyzing the situation." However, the VR technology and the way we integrated it had several drawbacks. In this discussion, we summarize the benefits and drawbacks that we encountered when integrating VR into teaching lean production.

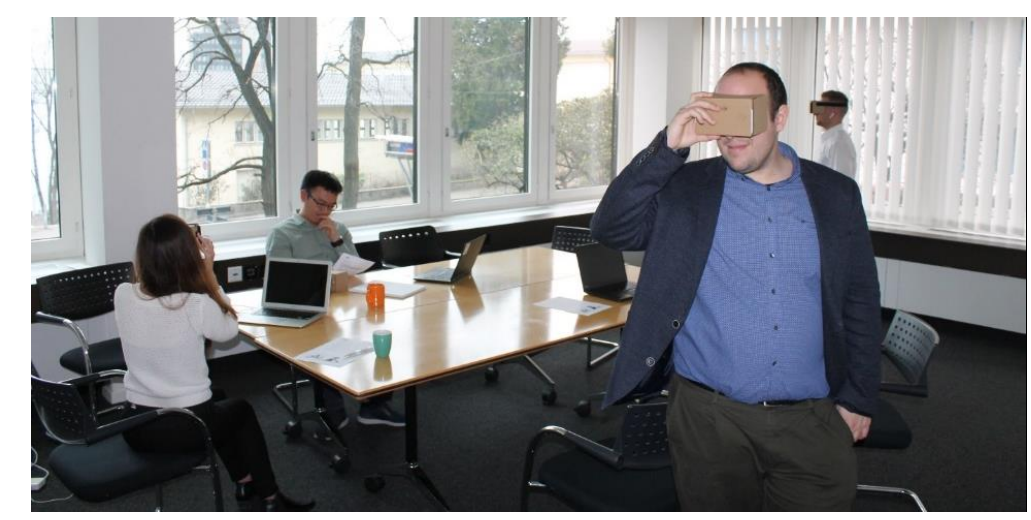

Fig. 3. Student group solving an assignment task with VR.

\subsection{Benefits}

With VR, students can explore factory environments whenever and wherever they like. One student reported, "It was good that you could discover the factory [at] your own speed." In contrast to factory tours, students can revisit the VR environment for any purpose, such as discussing content in groups. VR also allows the students to virtually visit multiple production sites in different locations and compare their differences without needing to travel. They can access areas and views that might be unavailable during a field trip, such as clean rooms and close-up views of machinery. VR also offers the possibility to blend different types of information, such as real images and videos of factory operations with overlaid digital information, to support students' learning experiences and learning outcomes.

One of the biggest advantages of VR is that it enables inquiry-based learning. One student remarked, "I think it's because it's more like an active discovery than like a passive [one]." While the questions guided students to seek certain answers, they also needed to use their own curiosity and intelligence when visiting the virtual environment and seeking the answers. One student asserted, "Since you have to look around, you start thinking . . . hey, so what am I actually looking at, or what should I be looking at?" 
Furthermore, the use of a modern technology, such as VR, is exciting for many students. This enthusiasm can improve their learning motivation. "Oh cool," commented one student. "We're doing something using VR. You know it's kind of a buzz wordit automatically excites you a little bit." Although the technology had a visible boost on student motivation, we did not find this excitement to last beyond the first explorations of the VR app.

\subsection{Drawbacks}

We also learned that the current state of technology has several drawbacks. For example, there is no way to ask questions during a virtual factory tour. It is also very hard to take notes or discuss with peers when using full-immersion VR glasses. Vision-impaired students using spectacles, found the VR glasses to be inconvenient. Also, in very few cases, we had students with older, incompatible smartphones or who experienced the app crashing. Some students who had experienced higher-end VR viewers, such as the Oculus Rift, were disappointed by the limited possibilities and low resolution offered by the cardboard variant.

The most serious drawback was that many students experienced motion sickness when using the VR app. To avoid this, several students reported that they stopped using the cardboard viewers and instead looked directly at the smartphone screen or used the computer. This workaround reduces the level of immersion, but it also affords the possibility to tour the virtual facility with others and take notes simultaneously.

\section{Conclusions}

VR can increase the effectiveness and efficiency of teaching lean production. In this paper, we showed how we integrated VR into classrooms for this purpose. We call this "Gemba VR." We also described all the details needed for other teachers to design similar assignments. In our experience, VR had mostly positive effects on students' learning experiences, as the immersion it offers can certainly improve learning outcomes. However, we also found that the current state of VR technology has some limitations and drawbacks that inhibit its use to teach all aspects of lean production.

A typical concern for teachers is that new technologies are expensive to access and difficult to master. In our experiences with the type of VR applications we used, those concerns do not hold water. Students can use technologies they already possess (smartphones), factory apps are offered for free, VR headsets are cheap, and their use is self-explanatory. A completely free alternative is to ask students to explore the 360degree videos on YouTube. With the rising improvement and availability of VR over the next few years, there is good reason to believe that its applications to lean teaching in higher education will only widen. In the near future, we hope to see an increasing availability of VR apps, such as the one provided by ABB. 
Acknowledgments. The authors are grateful for the financial support from Innovedum, the teaching innovation fund at ETH Zurich. We also want to thank the many companies that openly publish VR content from their factories online. Finally, we are indebted to the more than 200 students who have tested VR in our classes and provided informal and formal feedback on its applicability and opportunities.

\section{References}

1. Bicheno, J.: Kaizen and kaikaku. Manufacturing operations and supply chain management: the LEAN approach, pp. 175-184 (2001)

2. Mann, D.: Creating a lean culture: tools to sustain lean conversions. CRC Press (2014)

3. Candido, J.P., Murman, E.M., McManus, H.: Active learning strategies for teaching lean thinking. In: 3rd International CDIO Conference. Cambridge, MA, USA: www.cdio.org (2007)

4. Furlan, A., Galeazzo, A., Paggiaro, A.: Organizational and perceived learning in the workplace: a multilevel perspective on employees' problem solving. Organization Science, vol. 30, no. 2, pp. 280-297 (2019)

5. Scholten, K., Dubois, A.: Advancing the skill set of SCM graduates - an active learning approach. International Journal of Operations \& Production Management, vol. 37, no. 11, pp. 1683-1699 (2017)

6. McCall, M.W., Lombardo, M.M., Morrison, A.M.: Lessons of experience: how successful executives develop on the job. Simon and Schuster (1988)

7. Bicheno, J.: The new lean toolbox: towards fast, flexible flow. Production and Inventory Control, Systems and Industrial Engineering Books (2004)

8. Netland, T.H.: Critical success factors for implementing lean production: the effect of contingencies. International Journal of Production Research, vol. 54, no. 8, pp. 24332448 (2016)

9. Dede, C.: Immersive interfaces for engagement and learning. Science, vol. 323, no. 5910, pp. 66-69 (2009)

10. Sherman, W.R., Craig, A.B.: Understanding virtual reality: interface, application, and design. Elsevier (2002)

11. Burdea, G.C., Coiffet, P.: Virtual reality technology. John Wiley \& Sons (2003) 